\title{
Plane-wave decomposition of spatially random fields
}

\author{
Tan H. Nguyen, Hassaan Majeed, and Gabriel Popescu* \\ Quantitative Light Imaging Laboratory, Department of Electrical and Computer Engineering, \\ Beckman Institute of Advanced Science and Technology, University of Illinois at Urbana-Champaign, \\ Urbana, Illinois 61801, USA \\ *Corresponding author: gpopescu@illinois.edu
}

Received December 5, 2014; revised February 18, 2015; accepted February 18, 2015; posted February 20, 2015 (Doc. ID 228959); published March 24, 2015

\begin{abstract}
We investigate the uniqueness of the plane-wave decomposition of temporally deterministic, spatially random fields. Specifically, we consider the decomposition of spatially ergodic and, thus, statistically homogeneous fields. We show that when the spatial power spectrum is injective, the plane waves are the only possible coherent modes. Furthermore, the randomness of such fields originates in the spatial spectral phase, i.e., the phase associated with the coefficients of each plane wave in the expansion. By contrast, the spectral amplitude is deterministic and is specified by the spatial power spectrum. We end with a discussion showing how the results can be translated in full to the time domain. (c) 2015 Optical Society of America

OCIS codes: (030.1640) Coherence; (030.0030) Coherence and statistical optics.

http://dx.doi.org/10.1364/OL.40.001394
\end{abstract}

Optical fields encountered in practice fluctuate randomly in both time and space, and their behavior must be characterized through statistical quantities such as moments [1-3]. In imaging, because of field superposition [4], the second-order statistics is of particular interest. Field cross-correlation functions are measured whenever we study the relative properties of two fields, one of which is modified by a sample of interest, e.g., in interferometry, holography, and microscopy [5]. Field auto-correlation functions are used to describe the properties of the field itself [6], e.g., studying the statistics of the field upon propagation [7]. Spatial field correlations have been studied in the context of three-dimensional microscopy [6] and quantitative phase imaging [8].

Recently, it has been shown that a statistically homogeneous field can always be decomposed into a plane wave basis and, furthermore, its second order statistics is fully captured by a deterministic signal associated with the random field [9]. In this Letter, we study, for the first time to our knowledge, the conditions for which the plane wave set is unique. Previous reports addressed explicit solutions for the coherent modes by solving the integral equation for different source beams including the Gaussian Schell-model [10], twisted Gaussian Schell-model [11], Multi Gaussian Schell-beam model [12], partially coherent flatten Gaussian beam [13], anisotropic Gaussian Schell-model beams [14], partially coherent beams with optical vortices [15], and Besselcorrelated Schell-model beams [16]. The modal representations are also developed for three-dimensional sources including, for example, Lambertian sources [17], and spherical homogeneous sources [18]. For arbitrary fields, several theoretical and experimental methods have been proposed to determine the modes, see [19-21] and the references herein. The coherent mode calculations have been extended to electromagnetic fields, e.g., [22-24]. For a comprehensive review on the coherent mode decomposition and its application, see an article by Gbur and Visser [25]. Starikov studied the number of degrees of freedom and the number of uncorrelated variables of partially coherent sources [26]. Combining with the theory of angular spectrum representation for field propagation, Wolf derived several interesting properties for propagating coherent modes of the field from a bandlimited planar source [27].

In 1982, Wolf introduced the coherence mode decomposition. With this classic paper [28], it was shown that the cross-spectral density, $W\left(\mathbf{r}_{1}, \mathbf{r}_{2} ; \omega\right)=\left\langle U^{*}\left(\mathbf{r}_{1} ; \omega\right) U\left(\mathbf{r}_{2} ; \omega\right)\right\rangle$, of a stochastic field, $U$, can be represented in the form of Mercer's expansion as

$$
W\left(\mathbf{r}_{1}, \mathbf{r}_{2} ; \omega\right)=\sum_{n} \lambda_{n}(\omega) \phi_{n}^{*}\left(\mathbf{r}_{1} ; \omega\right) \phi_{n}\left(\mathbf{r}_{2} ; \omega\right),
$$

where the sum is uniform and absolute convergent. The angular bracket denotes the ensemble average over the spatially-varying ensemble. The functions $\phi_{n}$ are referred to as coherent modes. They are orthogonal eigenfunctions of $W$ corresponding to the eigenvalues $\lambda_{n}$, as defined by the Fredholm integral equation

$$
\int_{D} W\left(\mathbf{r}_{1}, \mathbf{r}_{2} ; \omega\right) \phi_{n}\left(\mathbf{r}_{1} ; \omega\right) \mathrm{d}^{3} \mathbf{r}_{1}=\lambda_{n}(\omega) \phi_{n}\left(\mathbf{r}_{2} ; \omega\right) .
$$

In Eq. (2), $D$ is the 3D spatial domain of interest, e.g., for a primary source, $D$ is a volume containing the source. Because $W$ is Hermitian, all the eigenvalues $\lambda_{n}$ are non-negative. Importantly, the random field itself, $U(\mathbf{r} ; \omega)$, can be expressed through the Karhunen-Loéve expansion using the same modes, as (see p. 66 in Ref. [1] $)$

$$
U(\mathbf{r} ; \omega)=\sum_{n} a_{n}(\omega) \phi_{n}(\mathbf{r} ; \omega) .
$$

Since $\phi_{n}(\mathbf{r} ; \omega)$ are deterministic functions, the randomness of $U(\mathbf{r} ; \omega)$ is contained in the coefficients, $a_{n}(\omega)$.

Let us consider a statistically homogeneous field at a certain plane of interest. Therefore, our coordinate is $\mathbf{r}_{1,2 \perp} \in D, D \subset \mathbf{R}^{2}$. The cross-spectral density simplifies to $W\left(\mathbf{r}_{1 \perp}, \mathbf{r}_{2 \perp} ; \omega\right)=W\left(\mathbf{r}_{2 \perp}-\mathbf{r}_{1 \perp} ; \omega\right)$ and Eq. (2) becomes

$$
\left[W \circlearrowright \phi_{n}\right]\left(\mathbf{r}_{\perp}, \omega\right)=\lambda_{n}(\omega) \phi_{n}\left(\mathbf{r}_{\perp}, \omega\right),
$$

where ( $)$ denotes the two dimensional convolution over $\mathbf{r}_{\perp}$. It can be shown that a set of plane waves, 
$\phi_{n}\left(\mathbf{r}_{\perp} ; \omega\right)=e^{i \mathbf{k}_{n \perp}(\omega) \cdot \mathbf{r}_{\perp}}$, satisfies Eq. (4) [9]. Using the property of the convolution with a complex exponential, we see immediately that $\left|W \oslash e^{i \mathbf{k}_{n \perp}(\omega) \cdot \mathbf{r}_{\perp}}\right|\left(\mathbf{r}_{\perp} ; \omega\right)=$ $\tilde{W}\left(\mathbf{k}_{n \perp} ; \omega\right) e^{i \mathbf{k}_{n \perp}(\omega) \cdot \mathbf{r}_{\perp}}$ with $\tilde{W}$ the Fourier transform of $W$. As a result, Eq. (4) yields the following eigenvalues

$$
\lambda_{n}(\omega)=\tilde{W}\left(\mathbf{k}_{n \perp} ; \omega\right) .
$$

Clearly, these eigenvalues are non-negative because $\tilde{W}$ is a (spatiotemporal) power spectrum.

Next, we show that the plane wave decomposition of $U$ is unique in the nondegenerate case, i.e., when the power spectrum, $\tilde{W}\left(\mathbf{k}_{\perp} ; \omega\right)$, is injective in the $\mathbf{k}_{\perp}$-space. Let us prove this claim by contradiction. Assume that $\tilde{W}$ is noninjective and, yet, allows for an eigenmode, $\phi_{n}\left(\mathbf{r}_{\perp} ; \omega\right)$, which is not a plane wave. It means that the Fourier transform of this eigenmode, $\tilde{\phi}_{n}$, is nonzero for at least two values of $\mathbf{k}_{\perp}$, say, and $\tilde{\phi}_{n}\left(\mathbf{k}_{\perp 1,2} ; \omega\right) \neq 0$. Taking the Fourier transform of Eq. (4), this condition implies

$$
\left(\tilde{W} \tilde{\phi}_{n}\right)\left(\mathbf{k}_{\perp 1,2} ; \omega\right)=\lambda_{n} \tilde{\phi}_{n}\left(\mathbf{k}_{\perp 1,2} ; \omega\right),
$$

which, after simplifying by $\tilde{\phi}\left(\mathbf{k}_{\perp 1,2} ; \omega\right)$, yields

$$
\tilde{W}\left(\mathbf{k}_{\perp 1} ; \omega\right)=\tilde{W}\left(\mathbf{k}_{\perp 2} ; \omega\right)=\lambda_{n}(\omega) .
$$

Since $\tilde{W}$ is injective, $\tilde{W}\left(\mathbf{k}_{\perp 1} ; \omega\right)=\tilde{W}\left(\mathbf{k}_{\perp 2} ; \omega\right)$ which implies $\mathbf{k}_{\perp 1}=\mathbf{k}_{\perp 2}$ therefore, contradicts our initial assumption of two distinct spatial frequencies. Thus, we proved that the plane waves are the unique mode decomposition of a statistically homogeneous field under the nondegeneracy case (injective $W$ ). There are many situations when this condition is not met in practice. For example, interference between multiple waves may generate power spectra that exhibit multiple maxima and minimums and are, thus, noninjective. A laser resonator is a common example whereby the spatial power spectrum is not injective and, as a result, the field can be expressed in other bases (e.g., Hermite-Gaussian polynomials). For the general case of noninjective $W$, one can partition the $\mathbf{k}_{\perp}$-space into disjoint sets $S_{\lambda_{1}}, S_{\lambda_{2}}, \ldots$ where $\lambda_{1}, \lambda_{2}, \ldots$ are nonzero values of the spatial power spectrum $\tilde{W}$. Each of these sets $S_{\lambda_{i}}$ corresponds to a subspace $T_{\lambda_{i}}$ of finite-energy signals whose spatial power spectrum is supported on $S_{\lambda_{i}}$. Then, by definition, the set of coherent modes can be chosen to be a union of complete orthogonal bases of all of these subspaces. The nondegeneracy is a special case of this solution where each disjoint set $S_{\lambda_{i}}$ contains exactly one wavevector and as a result, $T_{\lambda_{i}}$ only has a single plane wave as its basis.

In the following, we describe some implications of the plane wave decomposition of a spatially random field. Notwithstanding uniqueness, we can always expand a statistically homogeneous field, $U$, in a Karhunen-Loéve series Eq. (3) using plane waves as the coherent modes, $U\left(\mathbf{r}_{\perp} ; \omega\right)=\sum_{n} a\left(\mathbf{k}_{\perp n} ; \omega\right) e^{i \mathbf{k}_{\perp n} \cdot \mathbf{r}_{\perp}}, \quad$ where $a\left(\mathbf{k}_{\perp n} ; \omega\right)$ are random coefficients characterizing the ensemble of the spectral component $\tilde{U}\left(\mathbf{k}_{\perp n} ; \omega\right)$. It is worth clarifying the nature of this randomness. The coefficients $a\left(\mathbf{k}_{\perp n} ; \omega\right)$ do not depend on the time variable, $t$. The distribution of these coefficients is described by an underlying probability density for each mode $\mathbf{k}_{\perp n}$. For example, we can define an average, variance, etc., associated with the $\mathbf{k}_{\perp n}$ distribution of $a\left(\mathbf{k}_{\perp n} ; \omega\right)$. As emphasized early on by Wolf, the vast majority of fields encountered in practice are ergodic (see p. 345, in Ref. [28]). This allows us to replace the ensemble averages by averages over the measurable domain, i.e., space or time. Here, we employ spatial ergodicity to express $W$ in terms of a spatial average, namely

$$
\begin{aligned}
& W\left(\Delta \mathbf{r}_{\perp} ; \omega\right)=\left\langle U^{*}\left(\mathbf{r}_{\perp 1} ; \omega\right) U\left(\mathbf{r}_{\perp 1}+\Delta \mathbf{r}_{\perp} ; \omega\right)\right\rangle_{\mathbf{r}_{\perp 1}} \\
& =\sum_{m} \sum_{n} a^{*}\left(\mathbf{k}_{\perp m} ; \omega\right) a\left(\mathbf{k}_{\perp n} ; \omega\right) e^{i \mathbf{k}_{\perp m} \cdot \Delta \mathbf{r}_{\perp}} \\
& \times \int_{D} \mathrm{~d}^{2} \mathbf{r}_{\perp 1} e^{i\left(\mathbf{k}_{\perp m}-\mathbf{k}_{\perp n}\right) \cdot \mathbf{r}_{\perp 1}} \\
& =\sum_{m} \sum_{n} a^{*}\left(\mathbf{k}_{\perp m} ; \omega\right) a\left(\mathbf{k}_{\perp n} ; \omega\right) \\
& \times e^{i \mathbf{k}_{\perp m} \cdot \Delta \mathbf{r}_{\perp}} \delta^{(2)}\left(\mathbf{k}_{\perp m}-\mathbf{k}_{\perp n}\right) \\
& =\sum_{n}\left|a\left(\mathbf{k}_{\perp n} ; \omega\right)\right|^{2} e^{i \mathbf{k}_{\perp n} \cdot \Delta \mathbf{r}_{\perp}} \text {. }
\end{aligned}
$$

Therefore, taking the Fourier transform of Eq. (8), we find $\left|a\left(\mathbf{k}_{\perp n} ; \omega\right)\right|^{2}=\tilde{W}\left(\mathbf{k}_{\perp n} ; \omega\right)$, which is the spatial power spectrum of the field at wavevector $\mathbf{k}_{\perp n}$. We conclude that spatially ergodic fields can be decomposed into plane waves, whose amplitudes are deterministic, given by the spatial power spectrum, $\tilde{W}\left(\mathbf{k}_{\perp n} ; \omega\right)$. In other words, the spatial randomness in this case is only due to the spectral phase. Thus, spatially ergodic fields can be expressed quite generally as

$$
U\left(\mathbf{r}_{\perp} ; \omega\right)=\sum_{n} \sqrt{\tilde{W}\left(\mathbf{k}_{\perp n} ; \omega\right)} e^{i\left[\mathbf{k}_{\perp n} \cdot \mathbf{r}_{\perp}+\varphi\left(\mathbf{k}_{\perp n} ; \omega\right)\right]},
$$

where $\varphi\left(\mathbf{k}_{\perp n} ; \omega\right)$ is the spectral phase, the only random variable in the expansion. Note that the values of $\varphi\left(\mathbf{k}_{\perp n} ; \omega\right)$ do not affect the cross-spectral density, $W$, associated with $U$. In other words, we can construct a fictitious signal, referred to as the deterministic signal associated with a random field [9], $V$, by taking $\varphi\left(\mathbf{k}_{\perp n} ; \omega\right)=0$. Thus, $V\left(\mathbf{r}_{\perp} ; \omega\right)=\sum_{n} \sqrt{\tilde{W}\left(\mathbf{k}_{\perp n} ; \omega\right)} e^{i \mathbf{k}_{\perp n} \cdot \mathbf{r}_{\perp}}$ has the same spatial correlation, as the random field $U, \quad$ satisfying $\quad \int_{D} V^{*}\left(\mathbf{r}_{\perp 1} ; \omega\right) V\left(\mathbf{r}_{\perp 1}+\Delta \mathbf{r}_{\perp} ; \omega\right) \mathrm{d}^{2} \mathbf{r}_{\perp 1}=$ $W\left(\Delta \mathbf{r}_{\perp} ; \omega\right)$. In problems related to propagation of second-order correlations, it is much simpler to propagate the deterministic field to the plane of interest and then calculate its correlation, rather than propagating the correlation function itself.

We illustrate the relevance of the results presented here with two problems of spatial and temporal field distributions of common interest: coherent versus incoherent illumination in a microscope (Fig. 1) and, respectively, pulsed versus CW emission of a broadband laser (Fig. 2). Figure 1 illustrates how the randomness of the spectral phase controls the spatial field distribution. In microscopy, the spatial coherence of the illumination is governed by the numerical aperture of the condenser 
a)

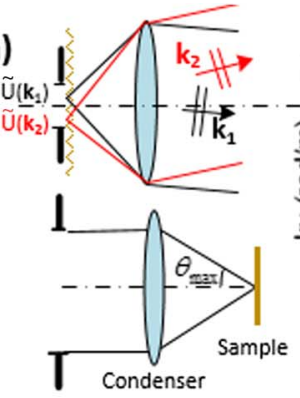

c)

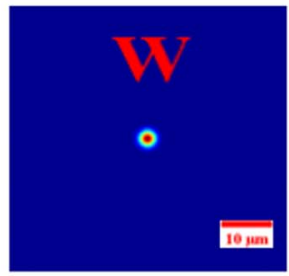

e)



g)



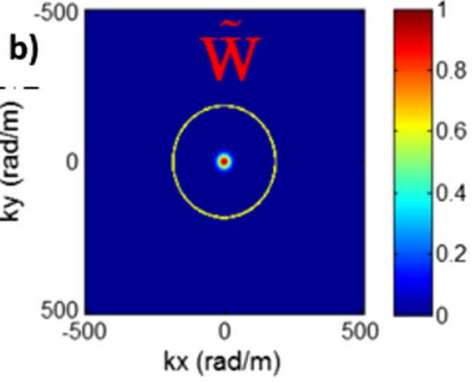

d)

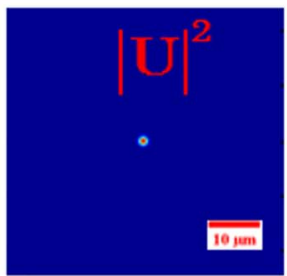

f)

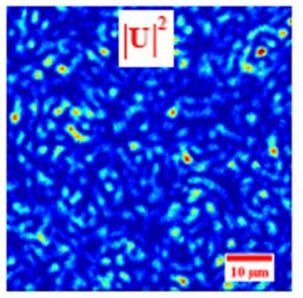

h)

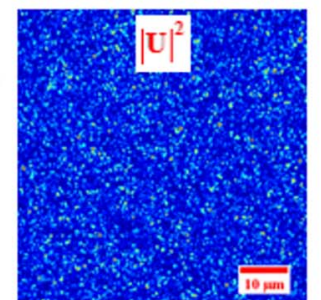

Fig. 1. (a) The role of the condenser aperture in controlling the spatial coherence of illuminating field in microscopy. (b) Simulated intensity image at the condenser's front pupil plane. This image is the spatial power spectrum of the illumination $\tilde{W}\left(\mathbf{k}_{\perp n} ; \omega_{o}\right)=\left|a\left(\mathbf{k}_{\perp n} ; \omega_{o}\right)\right|^{2}$. A Gaussian profile was used with the standard deviation given by $k_{0} \mathrm{NA}_{c}, \mathrm{NA}_{c}=0.09$ and $k_{o}=2 \pi / \lambda_{o}$ with $\lambda_{o}=532 \mathrm{~nm}$. The circumscribing circle denotes the diffraction limit circle $k_{0} \mathrm{NA}_{\text {obj }}$ assuming the objective has $\mathrm{NA}_{\mathrm{obj}}=1$. (c) Cross-spectral density, $W\left(\mathbf{r}_{1 \perp}-\mathbf{r}_{2 \perp} ; \omega\right)$, computed from the power spectral density $\tilde{W}\left(\mathbf{k}_{\perp n} ; \omega\right)$ in (b). (d) Intensity image of the focused field $U\left(\mathbf{r}_{\perp} ; \omega\right)$, obtained by zeroing the spectral phase $\varphi\left(\mathbf{k}_{\perp n} ; \omega\right)$. (e), (g) Simulated intensity images of the illumination field by using uniformly distributed in $[-\pi, \pi]$ of the spectral phase $\varphi\left(\mathbf{k}_{\perp n} ; \omega\right)$ and spectral amplitudes given by $\sqrt{\tilde{W}\left(\mathbf{k}_{\perp n} ; \omega\right)}$ in (b), for two different $\mathrm{NA}_{c}$ values 0.09 and 0.20 , respectively. (f), (h) Measured intensity images of the speckle obtained at the same imaging condition as indicated in bright field microscopy.

lens, $\mathrm{NA}_{c}=\sin \left(\theta_{\max }\right)$, assuming free space with refractive index of $n=1$. Each point in the front pupil plane of the condenser generates one mode for the illuminating field, $\left|a\left(\mathbf{k}_{\perp n} ; \omega\right)\right| \exp \left[i \mathbf{k}_{\perp n} \cdot \mathbf{r}_{\perp}+\varphi\left(\mathbf{k}_{\perp n} ; \omega\right)\right]$. The spectral phase distribution, $\varphi\left(\mathbf{k}_{\perp n} ; \omega\right)$, depends on the nature of the extended primary or secondary source (diffuser in the case of a microscope). Figure 1(a) illustrates how the coefficients $a\left(\mathbf{k}_{\perp n} ; \omega\right)$, are generated by an extended source in microscopy. Note each wavevector,
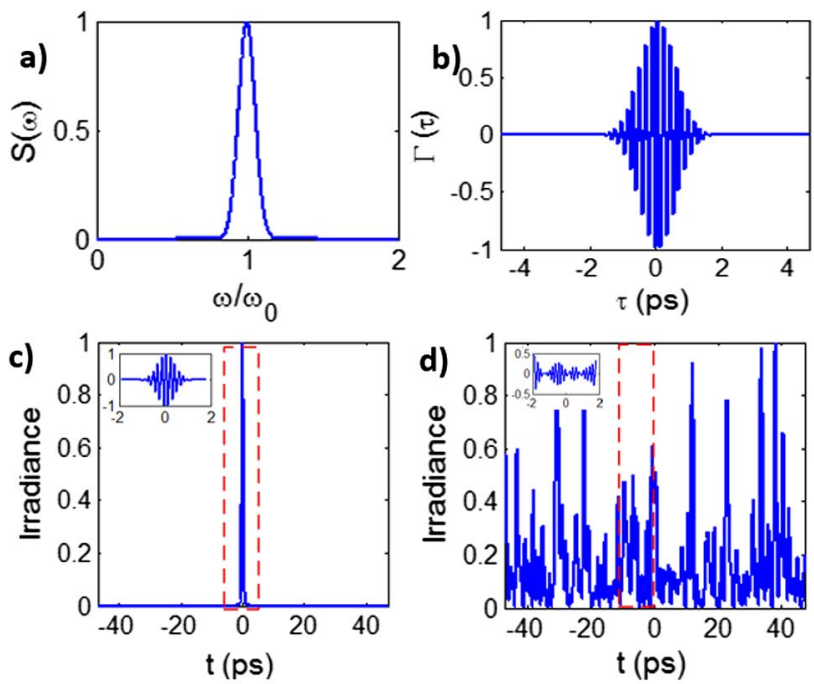

Fig. 2. (a) Simulated Gaussian power spectrum of a low-coherence source at central wavelength $\lambda_{o}=545 \mathrm{~nm}$, $\mathrm{FWHH}=30 \mathrm{~nm}$. (b) The temporal correlation function of the same source in (a) obtained by Fourier transforming the power spectrum. Envelop of this function is controlled by the bandwidth of the source. Larger bandwidth creates a more compact envelope and vice versa. The oscillation inside the envelope is given by the central optical frequency $\omega_{0}$. (c) Irradiance of a short pulse with the same correlation function in (b) generated by setting the phase value of 0 to the spectral component. The small inset shows the real part of the pulse around the region $t=0 \mathrm{ps}$ inside the dashed box. (d) Irradiance of a CW laser with the same correlation function in (b) with uniformly distributed spectral phase in $[-\pi, \pi]$. The small inset displays the real part of the continuous wave for the region specified by the dashed box.

$\mathbf{k}_{\perp n}$, corresponds to a different location on the extended source. The numerical aperture of the condenser determines how many components $\mathbf{k}_{\perp n}$ contribute to the total field $U\left(\mathbf{r}_{\perp} ; \omega\right)$. An intensity measurement over the area of this extended source gives the spatial power spectrum, $\tilde{W}\left(\mathbf{k}_{\perp n} ; \omega\right)$, as shown in Fig. 1(b). The 2D Fourier transform of the spatial power spectrum gives the crossspectral density $W\left(\mathbf{r}_{1 \perp}-\mathbf{r}_{2 \perp} ; \omega\right)$ shown in Fig. 1(c). As pointed above, the knowledge of the spatial power spectrum $\tilde{W}\left(\mathbf{k}_{\perp n} ; \omega\right)$ does not fully determine the coefficients $a\left(\mathbf{k}_{\perp n} ; \omega\right)$ but only their amplitudes, $\left|a\left(\mathbf{k}_{\perp n} ; \omega\right)\right|$. The phase of these coefficients defines the field distribution. Setting the phase of these coefficients to zero brings a tightly focused field $U\left(\mathbf{r}_{\perp} ; \omega\right)$, see Fig. 1(d). Meanwhile, uniformly distributed phase values from $[-\pi, \pi]$ generate the speckle pattern in Fig. 1(e), although the fields in these two cases have the same cross-spectral density $W\left(\mathbf{r}_{1 \perp}-\mathbf{r}_{2 \perp} ; \omega\right)$ shown in Fig. 1(c). Note that the waist of the focused beam in Fig. $1(\mathrm{~d})$ or the granularity of the speckle pattern ("speckle size") in Figs. $1(\mathrm{e})-1(\mathrm{~h})$ depend solely on the spatial power spectrum $W\left(\overline{\mathbf{k}_{\perp n}} ; \omega\right)$, which is itself controlled by the numerical aperture of the illumination. A smaller value of this numerical aperture gives a larger coherence area and a larger speckle size, see Figs. 1(e)-1(h).

The arguments can be translated in full to temporally random fields. Assuming a plane wave of wavevector $\mathbf{k}$ (spatially deterministic), we can show at once that, 
temporally, the field can be decomposed into monochromatic waves with random phases, namely

$$
U(\mathbf{k}, t)=\sum_{n} \sqrt{\tilde{W}\left(\mathbf{k}, \omega_{n}\right)} e^{i\left[\omega_{n} t+\varphi\left(\mathbf{k}, \omega_{n}\right)\right]} .
$$

If $\tilde{W}$ is an injective function on $\omega$, the monochromatic wave decomposition is unique. Figure 2 illustrates how the difference between a CW source and a light pulse of the same power spectrum is entirely in the distribution of the spectral phase $\varphi\left(\omega_{n}\right)$. We assume Gaussian power spectrum of central frequency $\omega_{o}$ [see Fig. 2(a)]. The corresponding temporal correlation function, $\Gamma(\tau)$, of this source is shown in Fig. 2(b). Figure 2(c) shows the irradiance of the field distribution when the spectral phase distribution is set to zero. When the spectral phase is randomly uniformly distributed over $[-\pi, \pi]$, while keeping the spectral amplitudes fixed, we obtain CW operation [Fig. 2(d)]. Both signals have the same correlation function as shown in Fig. 2(b). Therefore, not surprisingly, one method for obtaining short pulses, e.g., in Ti:Saph lasers, is referred to as mode-locking, which means converting the spectral phase from a random variable, $\{\varphi(\omega)\}$, to a deterministic function, $\varphi(\omega)$.

In sum, we studied the uniqueness property of the plane wave (and monochromatic wave) decomposition of statistically homogeneous (and stationary) fields. We showed that for the nondegeneracy case of the spatial-power spectrum $\tilde{W}$, plane waves are the only possible coherent modes. Similar conclusions can be extended for temporally random, stationary fields by replacing the spatial correlation function of a monochromatic field, $W\left(\mathbf{r}_{1 \perp}, \mathbf{r}_{2 \perp} ; \omega\right)$, with the temporal correlation function of a plane wave, $\Gamma\left(\mathbf{k} ; t_{1}, t_{2}\right)$, where $\mathbf{k}$ is the $3 \mathrm{D}$ wavevector of the plane wave. When ergodicity holds in both domains, space and time. For such cases, monochromatic plane waves will be the unique solution for the coherent mode decomposition problem when the spatialtemporal power spectrum $\tilde{W}\left(\mathbf{k} ; \omega_{n}\right)$ is injective, i.e., $\tilde{W}\left(\mathbf{k}_{n 1} ; \omega_{n 1}\right)=\tilde{W}\left(\mathbf{k}_{n 2} ; \omega_{n 2}\right)$ only when $\mathbf{k}_{n 1}=\mathbf{k}_{n 2}$ and $\omega_{n 1}=\omega_{n 2}$.

Combining the statistical homogeneity and spatial ergodicity condition, we proved that the spectral amplitudes are deterministic, and only the spectral phase is random. Although fields with strongly coherent spectral phase, e.g., all the spectral phase set to zero, and incoherent spectral phase have different intensity distributions, the second moment correlation is entirely the same.
This work was supported by the National Science Foundation CBET-1040461 MRI and Agilent Laboratories. For more information, visit http://light.ece.illinois .edu/.

\section{References}

1. L. Mandel and E. Wolf, Optical Coherence and Quantum Optics (Cambridge University, 1995).

2. J. W. Goodman, Statistical Optics (Wiley-Interscience, 1985), Vol. 567, p. 1.

3. R. J. Glauber, Quantum Theory of Optical Coherence: Selected Papers and Lectures (Wiley, 2007).

4. E. Abbe, Arch. Mikrosk. Anat. 9, 431 (1873).

5. G. Popescu, Quantitative Phase Imaging of Cells and Tissues (Mcgraw-Hill, 2011).

6. N. Streibl, J. Opt. Soc. Am. A 2, 121 (1985).

7. E. Wolf, Opt. Lett. 28, 1078 (2003).

8. T. H. Nguyen, C. Edwards, L. L. Goddard, and G. Popescu, Opt. Lett. 39, 5511 (2014).

9. T. Kim, R. Zhu, T. H. Nguyen, R. Zhou, C. Edwards, L. L. Goddard, and G. Popescu, Opt. Express 21, 20806 (2013).

10. A. Starikov and E. Wolf, J. Opt. Soc. Am. A 72, 923 (1982).

11. R. Simon, K. Sundar, and N. Mukunda, J. Opt. Soc. Am. A 10, 2008 (1993).

12. O. Korotkova, S. Sahin, and E. Shchepakina, J. Opt. Soc. Am. A 29, 2159 (2012).

13. F. Gori, M. Santarsiero, R. Borghi, and S. Vicalvi, J. Mod. Opt. 45, 539 (1998).

14. K. Sundar, N. Mukunda, and R. Simon, J. Opt. Soc. Am. A 12, 560 (1995).

15. S. A. Ponomarenko, J. Opt. Soc. Am. A 18, 150 (2001).

16. F. Gori, G. Guattari, and C. Padovani, Opt. Commun. 64, 311 (1987).

17. A. Starikov and A. T. Friberg, Appl. Opt. 23, 4261 (1984).

18. F. Gori and O. Korotkova, Opt. Commun. 282, 3859 (2009).

19. K. Kim and D.-y. Park, Opt. Lett. 17, 1043 (1992).

20. X. Xue, H. Wei, and A. G. Kirk, J. Opt. Soc. Am. A 17, 1086 (2000).

21. S. Flewett, H. M. Quiney, C. Q. Tran, and K. A. Nugent, Opt. Lett. 34, 2198 (2009).

22. F. Gori, M. Santarsiero, R. Simon, G. Piquero, R. Borghi, and G. Guattari, J. Opt. Soc. Am. A 20, 78 (2003).

23. J. Tervo, T. Setälä, and A. T. Friberg, J. Opt. Soc. Am. A 21, 2205 (2004).

24. C. Pask and A. Stacey, J. Opt. Soc. Am. A 5, 1688 (1988).

25. G. Gbur and T. D. Visser, Prog. Opt. 55, 285 (2010).

26. A. Starikov, J. Opt. Soc. Am. A 72, 1538 (1982).

27. E. Wolf, J. Opt. Soc. Am. A 3, 1920 (1986).

28. E. Wolf, J. Opt. Soc. Am. 72, 343 (1982). 